\title{
Parity-Violating Electron Scattering
}

\author{
Krishna S. Kumar for the MOLLER collaboration \\ Department of Physics and Astronomy, Stony Brook University, USA
}

DOI: http://dx.doi.org/10.3204/DESY-PROC-2014-04/255

\begin{abstract}
Measurements of parity-violating asymmetries in the scattering of longitudinally polarized electrons off unpolarized fixed targets are used to access leptonic and semi-leptonic weak neutral current amplitudes. One thrust of the current program is the measurement the elastic neutral weak amplitude at very low $Q^{2}$ from scattering off a heavy spinless nucleus, which is sensitive to the presence of a neutron skin. A second major thrust is the neutral current elastic amplitude at very low $Q^{2}$ off protons and electrons and in the DIS regime off deuterium, which allows precision measurements of the weak mixing angle at low energy and is thus sensitive to new physics beyond the Standard Model.
\end{abstract}

\section{Introduction}

The technique of electron scattering has been used for more than sixty years to study nuclear and nucleon structure using the experimental cleanliness and well-understood theory of electromagnetic interactions. Soon after parity-violation in the weak interactions was demonstrated, Zel'dovich noted that if an analogous neutral interaction existed, then parity violation would be manifested in longitudinally polarized lepton scattering off unpolarized nucleons due to the interference between the weak and electromagnetic amplitudes. He estimated that the parity-vioating asymmetry $A_{P V}$ would be of order $10^{-4} Q^{2}(\mathrm{GeV})^{2}$.

For typical fixed target experiments, $A_{P V}$ ranges from roughly $10^{-4}$ to as small as $10^{-7}$. Parity violation in deep inelastic electron nucleon scattering was first observed at SLAC [1], consituting an important test of the $\mathrm{SU}(2) \times \mathrm{U}(1)$ gauge theory of electroweak interactions. Over the past 30 years, the experimental techniques employed to measure these tiny left-right asymmetries have been steadily refined such that statistical and systematic errors better than 1 part per billion (ppb) are possible [2,3].With judiciously chosen targets and kinematics, this has facilitated measurements in several important physics topics, such as many-body nuclear physics, nucleon structure and searches for physics beyond the standard model at the TeV scale. In this review, we discuss the ongoing research program and future prospects.

\section{Neutron Skin of a Heavy Nucleus}

In a heavy nucleus such as ${ }^{208} \mathrm{~Pb}$, the fractional difference between neutron radius $R_{n}$ and proton radius $R_{p}$ is believed to be several percent. Analogous to the classic measurement of $R_{p}$ via elastic electron electromagnetic scattering, $R_{n}$ can be measured via parity-violating electron scattering [4]. The parity violating asymmetry in elastic scattering off a heavy spinless nucleus is proportional to the ratio of the neutron to proton form factors since the weak neutral current 
coupling of protons is much smaller than that of neutrons. Experimentally, there is some controversy as to how well $R_{n}$ is known [5]; the best guess is $\sim 5 \%$. A precise measurement of $R_{n}$ can have impact on nuclear theory, atomic parity violation [6] and neutron star structure [7].

With the improvements made on the control of helicity-correlated beam fluctuations, it became feasible to make a measurement of $A_{\mathrm{PV}}$ in elastic scattering off a ${ }^{208} \mathrm{~Pb}$ target. For a beam energy of $1.05 \mathrm{GeV}$ and a scattering angle of $6^{\circ}$ and $Q^{2} \sim 0.01 \mathrm{GeV}^{2}, A_{P V}$ is of order $0.6 \mathrm{ppm}$. A $3 \%$ measurement of $A_{P V}$ leads to a $1 \%$ measurement of $R_{n}$. A new experimental project known as PREX was approved and carried out a first data run at Jefferson Laboratory in 2010. The experiment ran for about 10 weeks in the spring of 2010. Statistics for a $9 \%$ $A_{P V}$ measurement and a $3 \% R_{n}$ determination was accumulated [8]. After corrections for beam fluctuations, the grand average was found to be $594 \pm 50$ parts per billion (ppb). After normalizing to the beam polarization and subtracting background, the PREX result is $A_{P V}=$ $656 \pm 60$ (stat) \pm 14 (syst) ppb.

The measured result corresponds to a value for the neutron skin of $R_{n}-R_{p}=+0.33_{-0.18}^{+0.16}$ $\mathrm{fm}$. While the result demonstrates that the neutron RMS radius is $2 \sigma$ larger than that of the protons the result is not yet precise enough to discriminate between various models based on mean field theory and other observables. A followup proposal to obtain the remaining statistics required for a $1 \% R_{n}$ measurement by the PREX collaboration has been approved and will likely run in 2016. Two other attractive nuclei to explore $R_{n}$ measurements are ${ }^{48} \mathrm{Ca}$ and ${ }^{120 / 124} \mathrm{Sn}$. In general low $Z$ nuclei tend to have a higher figure of merit due to the fact that the optimum $Q^{2}$ at which one must make the $A_{P V}$ measurement tends to increase, and the figure of merit rises with the square of the asymmetry times the count rate [9]. ${ }^{48} \mathrm{Ca}$ is particularly interesting because microscopic calculations may soon be feasible, which would allow $R_{n}$ to be related to poorly studied 3 -neutron forces. It turns out that ${ }^{48} \mathrm{Ca}$ is an ideal measurement at Jefferson Laboratory with a similar configuration to that used for PREX, except that the beam energy would be raised to $2.2 \mathrm{GeV}$, and an experimental proposal has recently been approved.

It is interesting to consider measurements of $\mathrm{Pb}$ and $\mathrm{Sn}$ at Mainz in the future, adapting the apparatus that would be required for a high precision proton weak charge measurement (see Sec. 3). It looks feasible to design the require apparatus for a $0.5 \% R_{n}$ measurement of ${ }^{208} \mathrm{~Pb}[10]$. The loss in rate due to the lower beam energy is roughly compensated by the larger available solid angle. The momentum resolution that would be required is about $1 \%$, and a system of baffles could be used to isolate elastic events while rejecting background from inelastics as well as from neutrals [11].

\section{Precision Weak Neutral Current Measurements}

Precision measurements of the properties of $\mathrm{W}$ and $\mathrm{Z}$ bosons and their couplings to leptons and quarks have allowed sensitive tests of the electroweak theory. No significant deviation from Standard Model predictions have been found. Nevertheless, experiments continue to probe for the indirect effects of new physics at the $\mathrm{TeV}$ scale by making more and more precise measurements of electroweak parameters. Weak neutral current (WNC) interactions at $Q^{2} \ll$ $M_{Z}^{2}$ can probe for heavy $\mathrm{Z}^{\prime}$ bosons or leptoquarks whose effects might be highly suppressed in measurements on the $\mathrm{Z}$ pole [12], and for dark sector mediators that have small admixtures to the $\mathrm{Z}$ boson [13]. Since the neutral current amplitude at the $\mathrm{Z}$ pole is imaginary, there are no interference terms with new, real four-fermion amplitudes. At low $Q^{2}$ on the other hand, interference effects might be measurable if sufficient accuracy is achieved $[2,3,14,15]$. 


\section{PARITY-Violating ELECTRON SCATTERING}

A general, model-independent way to parametrize the contributions of contact interactions of high-mass particles to low-energy measurements of electron scattering off target fermions $f$ is to use a Lagrangian [16] parametrized by coupling constants $g_{i j}(i, j=R, L)$, and a mass scale $\Lambda$. The goal of low energy neutral current measurements is to reach sufficient sensitivity to access $\Lambda / g_{i j} \geq 1 \mathrm{TeV}$ for as many different initial and final state fermions and chiral combinations as possible, similar sensitivity yet complementary to that of the highest energy colliders.

Over the past couple of decades, the three published measurements with sufficient sensitive to probe the $\mathrm{TeV}$-scale were the weak charge measurement in ${ }^{133} \mathrm{Cs}$ [17], the $\mathrm{NuTeV}$ neutrino deep-inelastic scattering measurement [18] and the measurement of $A_{\mathrm{PV}}$ in electron-electron (Møller) scattering [19] at SLAC. As we await the restart of the Large Hadron Collider (LHC) at full beam energy and design luminosity, improving these constraints and expanding their reach to include more fermion scattering combinations takes on increased significance. This is because such measurements will help narrow down the physics mechanisms that are responsible for any observed anomalies at the LHC.

The interactions of the Z-boson at low $Q^{2}$ can be approximated by four-fermion contact interactions. The parity-violating part of the electron-hadron interaction can then be given in terms of phenomenological couplings $C_{i j}$, where $C_{1 j}\left(C_{2 j}\right)$ gives the vector (axial-vector) coupling to the $j^{\text {th }}$ quark. In the Standard Model, all four couplings are functions of a single parameter: the weak mixing angle $\sin ^{2} \theta_{W}$. The atomic Cesium weak charge measurement measured one combination of $C_{1 u}$ and $C_{1 d}$ precisely.

At sufficiently forward angles and low $Q^{2}$, the hadronic structure uncertainty in the WNC elastic electron-proton amplitude becomes small enough such that one can measure the underlying coherent $2 u+d$ e-q amplitude combination to high precision, thus precisely constraining $2 C_{1 u}+C_{1 d}$. This combination is proportional to $1-4 \sin ^{2} \theta_{W}$. A $4 \%$ measurement of $A_{P V}$ would achieve a precision of $\delta\left(\sin ^{2} \theta_{W}\right)=0.0007$. This is the goal of the Qweak experiment [20] in Hall $\mathrm{C}$ at Jefferson Lab, which is reported on separately in these proceedings. The experiment has completed data-taking and published their first result based on commissioning data [21].

It would be highly complementary to obtain a new measurement of the proton weak charge with a new apparatus where the beam energy is smaller than $200 \mathrm{MeV}$. The dominant theoretical uncertainty in the prediction of $A_{P V}$ is due to higher-order radiative corrections involving $\gamma-Z$ box diagrams. In order to compensate for the lower energy, it will be necessary to obtain data over the full range of the azimuth using a solenoidal spectrometer [11]. Such an experiment would be a flagship measurement of the proposed new MESA facility at Mainz. A measurement of $A_{P V}$ with an ultimate goal of $2 \%$ overall error is compelling and seems feasible [22]

The upgrade of Jefferson Laboratory to $11 \mathrm{GeV}$ will allow precision measurements in parityviolating deep inelastic scattering (PV DIS). One measurement using a $6 \mathrm{GeV}$ beam and the existing high resolution spectrometers in Hall A has recently been published, and helped establish that the axial-quark couplings of light quarks to the $\mathrm{Z}$ boson are indeed non-zero [23]. A new dedicated high luminosity apparatus has been propopsed centered around a large superconducting solenoidal magnet [24]. This project, known as SoLID (Solenoidal Large Intensity Device), would allow $\sim 1 \%$ measurements of $A_{P V}$ for the first time over a range of $x$ and $Q^{2}$ values, as high as $x \sim 0.7$ ( $x$ is the fraction of the nucleon momentum carried by the struck quark). PV DIS measurements with such accuracy and kinematic range provides access to novel aspects of nucleon structure, such as charge symmetry violation and investigation of higher-twist effects. Most importantly, PV DIS allows the isolation of the linear combination $2 C_{2 u}+C_{2 d}$, which is difficult to measure using elastic scattering. $A_{P V}$ in DIS can be written as as functions of two parameters $a(x)$ and $b(x)$, which are functions of the parton distributions $f_{i}(x)$ and the elec- 
tromagnetic charges. For an isoscalar target such as deuterium, the dependence on structure largely cancels out in the $A_{P V}$ ratio of the weak and electromagnetic amplitudes.

The measurement would test the WNC amplitude in the lepton-quark sector, where there is currently a discrepancy with the theoretical prediction in the NuTeV result. Secondly, combined with other measurements in elastic electron-proton scattering, precise constraints would be possible on the lesser known axial-vector quark couplings $C_{2 i}$. This would, among other things, provide complementary constraints on various models with new heavy leptophobic $Z^{\prime}$ bosons [25] and leptoquarks [12]. More generally, the projected result for $A_{P V}^{D}$ of $0.6 \%$ translates into a measurement of a specific linear combination of the phenomenological couplings $C_{i j}$ to an accuracy of \pm 0.0083 , more than a factor of 30 better than the best current constraints. This translates into contact interaction scale reach $\gtrsim 2.5 \mathrm{TeV}$.

The Feynman diagrams for electron-electron (Møller) scattering involve both direct and exchange diagrams that interfere with each other. $A_{P V}$ for this process was first calculated in the late 70's [26] The electroweak theory prediction at tree level in terms of the weak mixing angle is $Q_{W}^{e}=1-4 \sin ^{2} \theta_{W}$; this is modified at the 1-loop level [27, 28] and becomes dependent on the energy scale at which the measurement is carried out, i.e. $\sin ^{2} \theta_{W}$ "runs". It increases by $\sim 3 \%$ compared to its value at the scale of the $Z^{0}$ boson mass, $M_{Z}$. The SLAC E158 experiment carried out the first measurement of parity violation in Møller scattering [19]. The grand average result for the parity-violating asymmetry in Møller scattering at $Q^{2}=0.03 \mathrm{GeV}^{2}$ was found to be: $A_{\mathrm{PV}}=-131 \pm 14$ (stat) \pm 10 (syst) (ppb).. From the measured result, and assuming that the only contributing chiral structure comes from the $\Lambda_{\mathrm{LL}}$ term, the $95 \%$ C.L. limit is $7 \mathrm{TeV}$ or $16 \mathrm{TeV}$ depending on the sign of the contact interaction term.

There is strong motivation to make further improvements in the accuracy of weak neutral current coupling constants at low energy. Improved measurements would keep pace with the improved sensitivity for discovery at the multi-TeV scale by experiments at the Large Hadron Collider. With the upgrade of Jefferson Laboratory to $12 \mathrm{GeV}$, a new project called MOLLER (Measurement of Lepton-Lepton Electroweak Reaction) is being designed to improve on the SLAC E158 measurement of the weak charge of the electron $Q_{W}^{e}$ by a factor of five. For the $2.3 \%$ total uncertainty envisioned, the sensitivity reach is $\frac{\Lambda}{\sqrt{\left|g_{R R}^{2}-g_{L L}^{2}\right|}}=7.5 \mathrm{TeV}$. The strongest constraints on four-lepton contact interactions come from LEP 2, approaching $5 \mathrm{TeV}$ for specific chiral combinations. However, the parity-conserving cross-sections and forwardbackward asymmetries studied at LEP 2 are blind to the parity-violating combination $g_{R R}^{2}-g_{L L}^{2}$ probed by MOLLER.

The prediction for MOLLER $A_{P V}$ is 35.6 parts per billion (ppb). The goal is to measure this quantity to a statistical precision of $0.74 \mathrm{ppb}$ and keep systematic errors to be significantly smaller, in order to achieve a $2.3 \%$ measurement of $Q_{W}^{e}$. MOLLER will greatly benefit from the steady improvement in the techniques employed to measure parity-violating asymmetries to sub-ppb systematic precision and to also achieve normalization control at the sub- $\%$ level. The MOLLER collaboration, a group of $\sim 100$ authors, is currently developing the technical design of the apparatus, and is in the process of seeking project funding of order $20 \mathrm{M} \$$ from IS DoE, NSF and international funding agencies. The goal is to obtain construction funding by 2017 so that experiment commissioning and first data collection can take place by 2020 . 


\section{PARITY-Violating Electron SCATTERING}

\section{Summary}

Parity-violating electron scattering is a mature field and addresses fundamental questions in a variety of different topics. The E158 experiment at SLAC has produced the most precise measurement of the weak mixing angle at low energy. The Qweak experiment at Jefferson Laboratory is on track to make an improved weak mixing angle measurement. The two future proposals at Jefferson Laboratory, SoLID and MOLLER, will lead to important new insights on the structure of the nucleon and yield new and more precise measurements of the weak mixing angle and constitute flagship projects after the $12 \mathrm{GeV}$ upgrade. It will be greatly beneficial to design and carry out a new measurement of the proton weak charge at Mainz that will improve on the Qweak experiment by a factor of 2 .

In parallel, it is greatly motivated to carry out new measurements of $A_{P V}$ on heavy nuclei to measure the ground-state neutron distribution. The PREX measurement is on-going and should produce a $1 \%$ measurement of the neutron radius in ${ }^{208} \mathrm{~Pb}$ in a few years. In the long run, it will be very important to produce comparable measurements using Ca and Sn isotopes at Jefferson Laboratory and at Mainz. Finally, the proposed new MESA facility at Mainz has the potential to add important new measurements to the impressive list of parity-violation experiments in order to probe nuclear and nucleon structure and to search for physics beyond the Standard Model.

\section{Acknowledgments}

This work has been funded by a grant from the Division of Nuclear Physics at the US Department of Energy's Office of Science.

\section{References}

[1] C.Y. Prescott, W.B. Atwood, R. Leslie Cottrell, H.C. DeStaebler, Edward L. Garwin, et al. Parity Nonconservation in Inelastic Electron Scattering. Phys.Lett., B77:347-352, 1978.

[2] K.S. Kumar, Sonny Mantry, W.J. Marciano, and P.A. Souder. Low Energy Measurements of the Weak Mixing Angle. Ann.Rev.Nucl.Part.Sci., 63:237-267, 2013.

[3] Jens Erler, Charles J. Horowitz, Sonny Mantry, and Paul A. Souder. Weak Polarized Electron Scattering. 2014

[4] T.W. Donnelly, J. Dubach, and Ingo Sick. Isospin Dependences in Parity Violating Electron Scattering. Nucl.Phys., A503:589, 1989.

[5] C.J. Horowitz, S.J. Pollock, P.A. Souder, and R. Michaels. Parity violating measurements of neutron densities. Phys.Rev., C63:025501, 2001.

[6] S.J. Pollock, E.N. Fortson, and L. Wilets. Atomic parity nonconservation: Electroweak parameters and nuclear structure. Phys.Rev., C46:2587-2600, 1992.

[7] C.J. Horowitz and J. Piekarewicz. Neutron star structure and the neutron radius of Pb-208. Phys.Rev.Lett., $86: 5647,2001$

[8] S. Abrahamyan, Z. Ahmed, H. Albataineh, K. Aniol, D.S. Armstrong, et al. Measurement of the Neutron Radius of 208Pb Through Parity-Violation in Electron Scattering. Phys.Rev.Lett., 108:112502, 2012.

[9] Shufang Ban, C.J. Horowitz, and R. Michaels. Parity Violating Electron Scattering Measurements of Neutron Densities. J.Phys., 39:015104, 2012.

[10] M. Thiel, D. Becker, M. Ferretti, K. Kumar, and C. Sfienti. Neutron skin studies of medium and heavy nuclei. EPJ Web Conf., 73:07007, 2014. 
[11] P.A. Souder and R. Holmes. New spectrometers for precision measurements of parity violation with polarized electrons. 1990.

[12] M.J. Ramsey-Musolf. Low-energy parity violation and new physics. Phys.Rev., C60:015501, 1999.

[13] Hooman Davoudiasl, Hye-Sung Lee, and William J. Marciano. Muon g-2, Rare Kaon Decays, and Parity Violation from Dark Bosons. Phys.Rev., D89:095006, 2014.

[14] Vincenzo Cirigliano and Michael J. Ramsey-Musolf. Low Energy Probes of Physics Beyond the Standard Model. Prog.Part.Nucl.Phys., 71:2-20, 2013.

[15] Jens Erler and Shufang Su. The Weak Neutral Current. Prog.Part.Nucl.Phys., 71:119-149, 2013.

[16] E. Eichten, Kenneth D. Lane, and Michael E. Peskin. New Tests for Quark and Lepton Substructure. Phys.Rev.Lett., 50:811-814, 1983.

[17] S.C. Bennett and Carl E. Wieman. Measurement of the $6 \mathrm{~S}$ to $7 \mathrm{~S}$ transition polarizability in atomic cesium and an improved test of the Standard Model. Phys.Rev.Lett., 82:2484-2487, 1999.

[18] G.P. Zeller et al. A Precise determination of electroweak parameters in neutrino nucleon scattering. Phys.Rev.Lett., 88:091802, 2002.

[19] P.L. Anthony et al. Precision measurement of the weak mixing angle in Moller scattering. Phys.Rev.Lett., 95:081601, 2005.

[20] D.S. Armstrong, A. Asaturyan, T. Averett, J. Benesch, J. Birchall, et al. The Qweak Experiment: A Search for New Physics at the TeV Scale via a Measurement of the Proton's Weak Charge. 2012.

[21] D. Androic et al. First Determination of the Weak Charge of the Proton. Phys.Rev.Lett., 111(14):141803, 2013.

[22] D. Becker, K. Gerz, S. Baunack, K.S. Kumar, and F.E. Maas. P2 - The weak charge of the proton. AIP Conf.Proc., 1563:78-81, 2013.

[23] D. Wang et al. Measurement of parity violation in electron-quark scattering. Nature, 506(7486):67-70, 2014.

[24] J.P. Chen, H. Gao, T.K. Hemmick, Z. E. Meziani, and P.A. Souder. A White Paper on SoLID (Solenoidal Large Intensity Device). 2014.

[25] Matthew R. Buckley and Michael J. Ramsey-Musolf. Precision Probes of a Leptophobic Z' Boson. Phys.Lett., B712:261-265, 2012.

[26] Emanuel Derman and William J. Marciano. Parity Violating Asymmetries in Polarized Electron Scattering. Annals Phys., 121:147, 1979.

[27] Andrzej Czarnecki and William J. Marciano. Electroweak radiative corrections to polarized Moller scattering asymmetries. Phys.Rev., D53:1066-1072, 1996.

[28] Jens Erler and Michael J. Ramsey-Musolf. The Weak mixing angle at low energies. Phys.Rev., D72:073003, 2005. 